\title{
Investigación sobre la especificidad de los efectos provocados en la morfogénesis uterina por la acción asociada de estrógenos y progesterona
}

\author{
Por R. MORICARd, F. MORICARd y S. Gothié \\ (Del Laboratorio de Altos Estudios de la Clínica Ginecológica del Hospital \\ Broca. París). \\ El presente trabajo fue cedido muy gentil- \\ mente por sus autores para su publicación \\ en Colombia, al profesor G. López-Escobar \\ durante su reciente estadia en el Hospital \\ Broca.
}

Se considera actualmente como establecido que el estudio de las biopsias de la mucosa endouterina, permite explorar la función luteal; sin embargo, ciertos resultados son aún discutidos. Desde el punto de vista práctico, el interés de este problema es considerable. Se puede admitir que por razón de la ausencia de especificidad de efectos de la acción progestínica sobre el epitelio vaginal, las modificaciones morfológicas cíclicas vaginales estudiadas por los frotis, no permiten explorar la acción progestínica. Trataremos de justificar en esta publicación la especificidad de los tests, o pruebas morfológicas uterinas utilizados en la exploración de la función luteal, es decir, de la acción asociada de estrógenos y progesterona. Expondremos sucesivamente: 1o Un estudio critico de los métodos preconizados para explorar la función luteal (biopsias, frotis, pregnandiol). $2^{\text {o }}$ Una descripción de los "tests" uterinos de la exploración cito-hormonal en la fase luteal. 3o Una interpretación critica de estos mismos "tests".

I. - ESTUDIO CRITICO DE LOS METODOS PROPUESTOS PARA EXPLORAR LA FUNCION LUTEAL

Cuatro métodos han sido preconizados. A.-Dosificaciones hormonales

Gracias a la realización de la dosificación de los estrógenos en la sangre (R. T. Frank 1925), o en la orina (Loewe, 1926; Zon- 
dek y Ascheim, 1927) parecía que la exploración de la función ovárica endocrina debiera hacerse por el dosaje de las eliminaciores hormonales urinarias. Más aún: Loewe ha emprendido la dosificación de la progesterona en la orina. En realidad la complejidad de las dosificaciones biológicas hormonales hacen estos métodos prácticamente inutilizables en la clínica corriente. Al presente, en Francia, varios autores han preconizado los dosajes hormonales como método de exploración de la función estrogénica y ciertos de ellos han permanecido como sus defensores.

En 1934 i| R. Moricard estudió comparativamente ciertas eliminaciones hormonales y la transformación deciduiforme uterina. Frente a la incertidumbre de estas dosificaciones él pensó si no sería preferible para explorar la actividad del cuerpo amarillo humano, determinar cuáles serian las modificaciones morfológicas especificas del receptor uterino bajo la acción de la progesterona.

\section{B.-Estudio del receptor uterino}

Ascheim en 1911 insistió sobre el interés del estudio de la carga en glucógeno del tracto genital de la mujer. Los trabajos de Ascheim olvidaron completamente el problema del tiempo de acción y de la especificidad progestínica de esta carga glucogénica de la mucosa uterina.

Es sabido que en 1921 Moukaye y Champy subrayaron el interés de ciertos adenomas benignos de tipo decidual en relación con los trastornos de la función del cuerpo amarillo.

En 1933, gracias a la realización industrial de las hormónas ováricas (Schering en Alemania, Roussell en Francia), fue posible provocar artificialmente el desarrollo del tracto genital de la mujer ovariectomizada $y$ definir las modificaciones morfológicas producidas por la acción única del estrógeno o por la acción asociada de estrógeno y progesterona. Kaufmann en 1933 fue el primero en lograr la aparición de un estado secretorio de la mucosa uterina en la mujer ovariectomizada por la acción de 300.000 unidades ratón de benzoato de estradiol asociado a 60 unidades coneja de progestina. Clauberg en la misma época obtenía por la inyección de progestina la transformación deciduiforme. En 1935 Moricard demostró que el estudio de las biopsias cervicales en la mujer castrada permitía reconocer los efectos morfogenéticos provocados por la acción de $30 \mathrm{mgm}$. de benzoato de estradiol (Proust, Moricard y Palmer). En 1936 estaba demostrado que el estudio de las biopsias endouterinas en las mujeres ovariecto- 
mizadas permitia reconocer la acción foliculinica aislada; en la "Revista Francesa de Ginecología", pág. 740, Moricard propuso el término: exploración cito-hormonal y describió el estado foliculinico y el esitado foliculo-iuteinico, que él definía así: tubo glandular "frangé" (franjeado), presencia de signos de actividad secretora del epitelio, estado disperso del vacuoma, mucicarminofilia del polo apical de la célula y presencia de glucógeno y presencia de espinas reticulares deformando la pared del tubo glanaular. Estos trabajos fueron reunidos en "Hormonologie Gynecolugique", tesis de Pierre Hoon, París, 1936.

Independientemente de estas investigaciones, Novak en 1935 había preconizado en los Estados Unidos una cureta que simplificó la toma endouterina, pero que no resolvió el difícil problema de la definición de los efectos provocados por la acción de las hormonas ováricas sobre la mucosa uterina de la mujer castrada, problema que Novak no abordó (ver conclusiones del "Texto de Ginecología" 41, 44, 48). Desde 1936 se ha estudiado sistemáticamente en el Laboratorio de la Clínica Ginecológica (Hospital Broca) la carga de glucógeno de la mucosa uterina como criterio de acción progestínica (Mocquot, Moricard, 1938). En los países cie lengua francesa Schockaert y Ferin en 1940, analizaron notablemente la acción de la pregneninolona sobre el útero de mujeres ovariectomizadas y precisaron las condiciones de aparición de la carga en glucógeno de la mucosa uterina. Ellos escribían: "Hemos utilizado el método de Best para poner en evidencia el glucógeno. Para Moricard su aparición a nivel del endometrio constituye el primer signo seguro de la acción de la progesterona. Hemos podido muchas veces comprobar la realidad de esta concepción. Sin embargo, según Engle (22), en la macaca el solo tratamiento foliculínico es suficiente para obtener células glandulares muy ricas en glucógeno. Nuestras búsquedas en las muestras tomadas en el curso del tratamiento foliculínico han sido siempre negativas mientras que la biopsia ejecutada después del tratamiento con pregneninolona, muestra bellos depósitos de glucógeno". Sobre este tema un trabajo de Bradbury y Brown publicado en "Clinical Endocrinology" en 1951, ha confirmado algunos de estos resultados sobre la transformación deciduiforme premenstrual.

En 1951 Funck-Brentano, Moricard y Robert, demostraron que en una hiperplasia quística secundaria a una implantación estrogénica en una mujer ovariectomizada, el glucógeno estaba 
ausente de la mucosa uterina. La carga en glucógeno de la mucosa uterina parece ser, pues, en la mujer, un criterio relativamente específico del equilibrio foliculo-luteínico, a pesar de que un trabajo publicado en 1950 por Master y Magallon pone en duda esta especificidad. En la mujer en menopausia no ooforectomizada, y bajo la acción de un tratamiento a la vez estrógeno y andrógeno, con dosis de 100 a $500 \mathrm{mgm}$. de propionato de testosterona en diez días, hay aparición de numerosas zonas claras basales que pueden ser interpretadas como equivalentes a una carga masiva en glucógeno. Este hecho necesita ser confirmado; sería preciso igualmente estar seguros de la pureza de los productos utilizados y sobretodo pensar en la acción ovárica de la testosterona por vía hipofisaria. En efecto, en 1941, R. y F. Moricard llamaron la atención sobre la activación producida por la testosterona en el desarrollo folicular de injertos ováricos. Por ctra parte en los tumores masculinizantes del ovario no hay carga en glucógeno de la mucosa uterina, y es más: en un caso hemos podido observar que la carga glucogénica vaginal disminuía. Se puede admitir que en las condiciones fisiológicas o patológicas comunes la carga masiva en glucógeno de la mucosa uterina es relativamente especifica de la acción estroprogestinica (foliculoluteinica) al obrar por espacio de algunos dias sobre una mucosa uterina foliculinizada.

Si la carga masiva en glucógeno es un test que permite reconocer precozmente la acción progestínica sobre un útero foliculinizado, esta carga en glucógeno tiene el enorme inconveniente de desaparecer bastante rápidamente. Ha sido preciso, pues, buscar tests morfológicos estables de la acción progestínica. Ellos han sido encontrados en las modificaciones conjuntivas de la pared de los tubos glandulares, y ya desde 1936 Moricard insistia sobre las modificaciones del retículo peritubular. Este estudio ha logrado aislar como test de acción foliculoluteínica, la presencia de "espinas conjuntivas". Estas espinas son el homólogo del encaje uterino de pseudogestación descrito en la coneja por Bouin y Ancel en 1911. Universalmente es conocido que fue la utilización de este test lo que permitió a Corner y willard Allen aislar en 1929 la progestina que los químicos llamaron progesterona (Allen, Butenandt, Fels, Slotta. 1934) - El estudio de las modificaciones conjuntivas del corion citógeno de la mucosa uterina humana, es pues, una aplicación directa de la histofisiología experimental. A raíz de los trabajos de Daron y Barthelmez, y desde 1938 (tesis 
de Bourgugnon) se han estudiado sistemáticamente en el Hospital Broca la diferenciación de las arterioles espirales en la mucosa uterina humana. Esta actitud es totalmente diferente de la de Noyes, Hertig y Rock, quienes en 1950 escribían en su impor-, tante trabajo: "Dating the endometrial Biopsy" lo siguiente: "Not all variations that occur in the endometrium are useful for dating. For example, the tortuosity of the endometrial glands and the coiling of the spiral arterioles increase so gradually that quantitative differences from day to day cannot be detected". Fara lograr un método de exploración de la función luteal el problema para nosotros no está en fechar cronológicamente el aspecto de la mucosa uterina, sino en establecer el determinismo hormonal de un aspecto fácilmente reconocible y dependiente específicamente de la acción de la progesterona. A este respecto la diferenciación de las espinas conjuntivas y de las arteriolas espirales xuede ser considerada como uno de los tests específicos del equilibrio foliculo-luteinico. Estas diferenciaciones conjuntivas tienen, pues, un interés capital como base de exploración de la funsión luteal. Es preciso recordar que el estudio biológico de la morfogénesis uterina experimental ha permitido aislar seis grupos de factores, a saber: 1 o La naturaleza de la sustancia actuante; $20 \mathrm{La}$ dosis; $3^{\text {o }} \mathrm{La}$ asociación folículo-progestínica u otras; 4ㅇ La duración de la acción de las hormonas; 5 o La cronología de las acciones hormonales; 6 ? Las cualidades locales de los receptores uterinos - vaginales a la acción progestínica, la cual varía con el grado previo y actual de su foliculinización. Experimentaimente, la progesterona puede estar presente en la circulación, sin por ello provocar la formación del encaje uterino en la coneja. Desde el punto de vista biológico, R. Moricard en 1934-35 confirmó a este respecto los trabajos de Corner y de Clauberg, quienes demostraron que la progesterona podia actuar sobre el útero de la coneja impúber sin formar el "encaje uterino", y en 1936 él mismo introdujo la noción de la receptividad hormonal condicional del útero a la acción progestínica. Esta noción se aplica también al receptor vaginal. Este dato esencial obliga a no considerar como significativos en la mujer sino los resultados positivos, es decir, aquellos en los que la biopsia uterina muestra una carga masiva en glucógeno, o mejor una diferenciación de las arteriolas espirales, muy comúnmente asociada a la de las espinas conjuntivas. El enorme inconveniente de la exploración de la función luteal, por el estudio de la mucosa uterina es que precisa una biopsia 
y por lo tanto, lógicamente se ha buscado si el estudio de extendidos vaginales daría en la mujer un criterio cierto de la acción progestínica, haciendo así inútil esta biopsia.

C.- La utilización de los frotis vaginales en la exploración luteal

Stockard y Papanicolau en 1927, Evans y Long en 1921, Allen en 1922, determinaron el ciclo vaginal de los roedores. Este ciclo fue luego estudiado en la mujer por Papanicolaou. Desde 1936 se ha reconocido que la castración provoca en la mujer un aspecto característico de los frotis (coloreados a la hematina-eosina) reconocible por la presencia de células de descamación de forma ovalada, con núcleo relativamente voluminoso y asociadas a numerosos polinucleares (Papanicolau y Shorr 1936, Salmón y Geist 1936). En 1943 Madame Moricard página 129, en "Hormonologie Sexuelle Humaine" insistía sobre estos resultados. Sabido es que desde 1942 las publicaciones de Papanicolaou tenian por base un nuevo método de coloración tricroma de los extendidos. Esto permitió caracterizar muy bien la acción del estrógeno sobre la vagina y puso en evidencia el aumento del índice acidofílico bajo la influencia de la acción estrogénica. La caracterización de la acción estrogénica sobre la vagina de la mujer es, pues, un hecho bien establecido. Papanicolaou tuvo el gran mérito en sus publicaciones de no concluir jamás que el estudio de los frotis vaginales permitia reconocer de manera especifica la acción progrestinica. Ciertos anatomopatólogos y algunos médicos especializados en la colpocitología han querido ver en una descamación en grumos (placas), de células plegadas de predomìnio basófilo, un criterio específico de la acción progestínica. Moricard ha luchado contra esta interpretación que cree errónea, pues este aspecto colpocitológico se observa tanto en la fase luteal como en el caso de descenso de la acción estrogéníca en la mujer castrada. Este aspecto de células plegadas en grumcs no es pues especifico de la acción progestinica. Esta esencial e importante anotación explica por qué en 1950 Ferin y Demol, en 1951, Albeaux-Fernet, Busser y Zerah, en 1952, Gaudefroy y Triquet, llegaron a la conciusión de que los frotis vaginales no permiten explorar de manera segura la función iuteal. En 1952 Pundel en la segunda edición de su libro sobre los frotis vaginales endocrinos, subraya el hecho importante de que el plegamiento celular aislado puede ser producido únicamente por la caida de la foliculina y añade: "En este caso las células permanecen por lo general aisladas" (el subrayado es nuestro). Pundel con- 
cluye a propósito de este plegamiento en grumos: No queremos afirmar la especificidad absoluta de esite signo. Se puede, pues considerar como establecido actualmente que los frotis vaginales no presentan en la mujer una imagen especifica de la acción progestinica, asi se repita el frotis vaginal cuotidianamente. Esta conclusión resume la opinión de los biólogos, quienes se han visto todos obligados a recurrir exclusivamente a la mucosa uterina para dosificar la progesterona. Si la simple ejecución de un extendido vaginal hubiera allegado un test específico de la acción progestínica, la expioración funcional ginecológica se hubiera revolucionado; esta revolución sin embargo no se ha procucido.

D.-La dosificación de los metabolitos ce la progesterona en la exploración de la fase luteal

Si las dosificaciones de la progesterona en la orina se muestran prácticamente imposibles. ¿no se prodria buscar en la orina un metaboiiic caracteristico de la progestercna? Se llegaría así a evitar una biopsia endouterina en la exploración de la función luteal. Es sabido que Butenandt trabajando sobre la orina de la mujer en cinta aisló en 1928 el pregnandiol. En 1937 Wenning y Brown, alumno de Butenandt, estudiaron en forma notable la eliminación de este metabolito en el embarazo normal y patológico. Marrian en Inglaterra, en Francia Simonnet y Robey, Jayle y sus colaboradores, extendieron y precisaron estos resultados. Esquemáticamente en la fase luteal la cantidad de pregnandiol eliminado es aproximadamente de $5 \mathrm{mgm}$. por litro, y en la fase folicular es por lo general inferior a $1 \mathrm{mgm}$ por litro de orina. En esta dosificación se está, pues, en el límite de la sensibilidad técrica de los métodos. Por otra parte, si en la mujer el pregnandiol urinario tiene un origen predominantemente ovárico, otra parte proviene de la suprarrenal. Una buena porción de la progesterona no se elimina bajo la forma de pregnandiol en la orina sino que pasa en parte bajo la forma de pregnenolona. A pesar de todo y de la poca certeza del metabolismo hepático y renal intermediario de los metabolitos de la progesterona, seria evidentemente muy práctico llegar a la exploración de la función luteal por el dosaje bioquimico de ciertos metabolitos urinarios, si este dosaje fuera a la vez simple, especifico y cuantitativo de la carga humoral en progesterona. Jayle y colaboradores han estudiado muy bien este problema y por razón de las múltiples dificultades encon- 
tradas, Jayle ha debido modificar a menudo sus técnicas habiendo llegado a dar, definitivamente al parecer, la preferencia a una dosificación colorimétrica de ciertos esteroides urinarios elimiriados bajo la forma de glucoronidatos y que son en su mayoría metabolitos de la progesterona, pero la especifidad de origen de estos metabolitos no está aún establecida. Por razones de simplificación técnica y de especificidad relativa de la reacción de ab sorción en lo que se refiere al pregnandiol, en el laboratorio de la Clínica Ginecológica de Broca se utiliza la técnica de Huber y Borth (absorción sobre alúmina) puesta al día en el Servicio de Wateville en Ginebra. Esta técnica ha sido utilizada en el laboratorio de la Clínica por R. Moricard y S. Gothié. Aunque delicada ella es relativamente simple y lo bastante específica para el pregnandiol, pero no permite la dosificación de la pregnenolona; lo que no parece en realidad muy importante para la exploración de la función luteal fuera de la gestación. Hechos reunidos por Mademoiselle Gothié parecen demostrar una correlación notoria entre la eliminación del pregnandiol dosificado por el método de Wateville-Borth y los tests morfológicos uterinos de acción progestínica, tales como han sido establecidos por Moricard.

Estudio comparado de dosificaciones simultáneas a las

biopsias de endometrio

Se practicaron 48 biopsias al mismo tiempo que se hacía la dosificación del pregnandiol. En 21 mujeres no había signo característico en la mucosa uterina de acción progestínica, en una sola de ellas se encontró pregnandiol en la orina, pero no había sido encontrado el día anterior; la biopsia había sido hecha en la mañana del "decalaje" térmico.

En los 27 casos en los que la mucosa estaba en estado foliculoluteínico, se encontró eliminación de pregnandiol. Cabe anotar sin embargo un caso en el que se encontró una diferenciación mínima de las arteriolas espirales sin pregnandioluria dos días antes de la biopsia.

Existe pues en la mujer normal una concordancia notoria entre la tasa de pregnandiol urinario y las modificaciones histológicas de la mucosa uterina.

Exploración de ciclos normales

Hemos estudiado 13 ciclos en nueve mujeres normales. En seis de ellas se pudo practicar una biopsia de endometrio. El 
pregnandiol apareció en la orina inmediatamente después del "decalaje" térmico; en dos casos lo precedió por algunas horas. La aparición es por lo general muy repentina y brutal (de 3 a 8 mgm 0/00); la concentración oscila entre 2 y $9 \mathrm{mgm}$. en el curso de la fase luteal, la caída brusca premenstrual puede producirse dos días antes de la regla, o bien persistir la concentración hasta la hemorragia menstrual; no hemos encontrado pregnandiol durante la regla. La eliminación total de pregnandiol en la orina es de 20 a $60 \mathrm{mgm}$. durante toda la fase luteal que dura 8 a 12 días. Cuando las dosificaciones son practicadas cuotidianamente se comprueba en el curso del ciclo, la ausencia de pregnandiol en una o dos muestras. De manera general esta ausencia está precedida o seguida por una alta concentración. Las biopsias tomadas en el momento de la aparición del pregnandiol no tienen ninguna de las características que hemos considerado como criterio de la acción foliculoluteínica.

El estado foliculoluteínico con carga masiva en glucógeno aparece del segundo al tercer día de la eliminación de pregnandiol, el edema del corion del cuarto al quinto día, las arteriolas espirales y las espinas conjuntivas del quinto al doceavo día.

El aspecto de la mucosa parece estar mucho más en relación con la duración de eliminación del pregnandiol que con la cantidad eliminada de éste.

En una hepática las cantidades eliminadas permanecieron bajas; cantidad total eliminada $10 \mathrm{mgm}$. Talvez en este caso debiera tenerse en cuenta el metabolismo intermediario hepático.

En dos casos en que el ciclo era largo - 34 a 40 días- no se encontró pregnandiol en la orina sino 8 a 10 días antes de las reglas. La curva térmica por lo general difásica, las biopsias tardias son foliculoluteínicas.

Hemos dosificado el pregnandiol urinario en dos mujeres que sufrieron una histerectomía con conservación de ovarios: LAU: operada dieciséis dias después de la regla, mucosa uterina en glucógeno masivo, la mañana siguiente a la intervención se encuentran $6 \mathrm{mgm}$. de pregnandiol y la eliminación decrece rápidamente para anularse al cuarto día; excreción total de $10 \mathrm{mgm}$.

GUIL: operada quince días después de las reglas, mucosa uterina en el comienzo de formación del espacio claro basal, eliminación de pregnandiol sólo duró dos días; excreción total: 8 mgm. 
BC: operada seis dias después de las reglas, no se pudo poner en evidencia el pregnandiol en el transcurso posterior a la intervención.

\section{E.- Problema práctico de la escogencia de un método para ex- plorar la función luteal}

Desde el punto de vista investigativo, se pueden comparar a la vez los frotis, las biopsias y las eliminaciones urinarias, llegando aun a cristalizar y definir químicamente las sustancias eliminadas, lo que por lo general no se hace. Pero cuando se trata de hacer diagnósticos de rutina y de tratar los enfermos, para el médico práctico el problema es muy diferente $y$ es preciso escoger un método simple y especifíco que permita explorar la función luteal; el director de un laboratorio de una clínica universitaria que rehuse dejarse sumergir por trabajos estériles de rutina, debe saber escoger un método que le permita explorar ia función luteal ce manera signïficativa, sin que necesite de un estudio matemático estadístico delicado para poder interpretar los resultados.

En la exploración de la función luteal 19 el frotìs vaginal: es éste el único que debe ser descartado. En efecto, los frotis exploran la función estrogénica y no la acción progestínica. La caída de la acción estrogénica o la aparición de la progestina dan sencillamente el mismo frotis. 20 La dosificación del pregnandiol urinario puede ser utilizada pero la técnica es en realidad larga y delicada y está por encima de las posibilidades de un laboratorio corriente no especializado. Tiene la ventaja de evitarle a la enferma la toma endo-uterina. Un solo técnico puede ejecutar al día alrededor de seis dosajes y como es preciso hacer al menos coos dosajes por ciclo, se pueden explorar tres enfermas al día. Esto significa generalmente gastos por encima de la práctica corriente. $3^{\circ}$ En la práctica la biopsia endouterina es el método que se debe preconizar: el inconveniente es la necesidad de una toma endouterina que sin embargo es muy fácil para el ginecólogo adiestrado. Un técnico especializado puede ejecutar al día alrededor de quince biopsias, la lectura necesita un patólogo de buen entrenamiento, pero ésta lectura puede hacerse fácilmente en menos ve una hora. Una sola biposia practicada algunos dias antes de la menstruación permite si ella es positiva reconocer la existencia de la acción progestínica. Explorar en seis horas de trabajo tres o quince enfermas es un argumento importante. Pero 
sobretodo la biopsia endouterina aporta tests específicos de la acción progestínica.

\section{Ir.- TESTS DE EXPLORACION CITO-HORMONAL EN LA FASE LUTEAL}

Nos limitaremos en este capitulo a los tests que sirven de base para la interpretación de las biopsias de rutina.

En trabajos anteriores, R. Moricard comparó el ciclo uterino normal con el estado de los ovarios; provocó la morfogénesis artificial del útero y la vagina en la mujer ovariectomizada y siguió las modificaciones del tracto genital en mujeres estériles con trompas no permeables, pero cuyos ovarios eran normales. En el curso de operaciones realizadas por Palmer, $R$. Moricard y De Senarclens, pudo examinar algunas piezas operatorias de ruptura folicular muy reciente y estudiar simultáneamente la mucosa uterina.

Para los exámenes de rutina las biopsias se han fijado en Bouin alcohólico y se han coloreado al Picropunzó de Curtis (Van Gieson modificado), y con la goma iodada para poner en evidencia el glucógeno. Para los frotis vaginales se ha utilizado el $\mathrm{Pa}-$ panicolaou. Con el objeto de simplificar esta descripción, de una manera sistemática se puede considerar el ciclo uterino de veintiocho días, colocando la ovulación a los catorce días. No nos detendremos en las investigaciones realizadas sobre el retículo (coIoración de Bilchowsky) sobre el aparato de Golgi (fijación de Da Fano) sobre las fosfatasas (reacción de Gomori), sobre la mucina (muco-carmin de Mayer), sobre los ácidos nucleicos (reacción de Feulgen o de Brachet). No mencionaremos tampoco los interesantes trabajos de Champy y Cuajard sobre el sistema nervioso terminal o de Vokaert sobre la histofotometría uterina.

\section{Estado foliculinico}

Corresponde al desarrollo folicular comprendida también la ruptura folicular con el comienzo de la penetración vascular por la granulosa. La función uterina esencial es en este estado, permitir el ascenso espermático seguido eventualmente de fecunclación intratubaria. Desde el punto de vista cronológico en un ciclo de veintiocho días y en el que la ovulación se haga a los catorce días, el estado foliculínico va esquemáticamente hasta los quince días del ciclo. 
a) Estado foliculinico correspondiente al desarrollo folicular está caracterizado en la mucosa uterina por tubos glandulares en vía de proliferación; el citoplasma de las glándulas uterinas es basófilo, los núcleos son elipsoidales. En este epitelio la parte periférica de los núcleos está en contacto con la pared de los tubos glandulares, el glucógeno generalmente está ausente y si existe está reducido a algunos raros granos situados en la base del epitelio y no ocupa más que la décima parte de la célula epitelial y sólo en algunos tubos. Las mitosis son numerosas. Las paredes de los tubos glandulares en la parte superficial del corion son rectilíneas o ligeramente enrolladas en hélice; esta disposición no debe confundirse con las espinas conjuntivas que no están cliferenciadas aún. En el corion débilmente edematoso se pueden encontrar algunas células en mitosis y no hay arteriolas espirales.

b) Estado foliculinico correspondiente a la ruptura folicular: conserva caracteres análogos, no hay carga masiva en glucógeno en los tubos glandulares, y si hay glucógeno en algunos tubos permanece poco abundante. Los núcleos elipsoidales están aún en contacto con la pared de los tubos glandulares. Para observar este aspecto uterino, es necesario que en el folículo roto la granulosa no haya sufrido aún una transformación luteínica completa; hay solamente un principio de penetración vascular y la pared folicular está entonces aplastada. En el momento de la ruptura folicular la acidofilia vaginal pasa por un máximo de a.jrededor de $50 \%$ o más, de células acidófilas. El estado foliculinico puede ser realizado en la mujer ovariectomizada por la inyección de $30 \mathrm{mgm}$. de benzonato de estradiol repartidos en un período de diez a quince días. Para provocar la secreción cervical y la acidofilia vaginal una dosis de $5 \mathrm{mgm}$. repartida en cinco días es ampliamente suficiente. La progesterona o la testosterona, inyectadas solas parecen no ser capaces de reproducir artificialmente estas modificaciones uterinas o vaginales. El determinismo foliculínico de esta morfogénesis está, pues, definitivamente establecido. Estos aspectos morfológicos son lo suficientemente característicos para servir de base a la exploración de la función estrogénica. Es posible que en la mujer una débil secreción de progesterona preceda o acompañe la ruptura folicular pero la acción de la progesterona no es reconocible de manera segura sobre la mucosa uterina en el momento de la ruptura folicular; es por ésta razón que hemos hecho entrar la evolución en el "estado foliculínico". 


\section{Estado foliculo-luteinico}

Corresponde a la actividad del cuerpo amarillo que habitualmente sigue a la ovulación. La función uterina esencial es permitir la nidación del huevo. Desde el punto de vista cronológico va de dieciséis a dieciocho días. El determinismo hormonal de la morfogénesis uterina es a la vez foliculínico y progestínico. Cronológica y morfológicamente el estado foliculoluteínico puede subdividirse así:

a) Es.tado foliculo-luteínico de glucógeno masivo, cuerpo amarillo funcional, a los diecisiete días la zona clara basal regularmente formada ocupa aproximadamente el tercio externo de las células epiteliales La topografía del glucógeno varía con el grado de penetración del fijador alcohólico. Una parte del glucógeno, tiene así una topografía apical y parece excretado en la luz de los tubos glandulares. La carga masiva en glucógeno (1) se produce aproximadamente 48 horas después de la ruptura folicular, cuando la granulosa está ya bien penetrada por los vasos y muestra la presencia de lípidos. Cuando la carga masiva en glucógeno existe, no hay ya mitosis en el epitelio, poco o ningún edema en el corion y las espinas conjuntivas y arteriolas espirales no se han diferenciado aún.

La carga masiva en glucógeno puede ser obtenida sobre un útero foliculinizado, con la inyección de $100 \mathrm{mgm}$. de progesterona y es visible después de dos o tres días de comienzo de la acción progestínica. La inyección aislada de estrógeno no permite realizar esta carga masiva en glucógeno, así haya una sobrecarga estrogénica (Funck-Brentano, Moricard y Robert). No parece tampoco que en las condiciones fisiológicas y patológicas babituales la testosterona pueda intervenir fisiológicamente en esta carga masiva en glucógeno, que es uno de los primeros efec-

(1) En 1936, no se sabía si la carga masiva en glucógeno precedía, acompañaba o seguía a la formación del cuerpo amarillo. Por esta razón se había designado la carga masiva en glucógeno con el nombre de "estado foliculínico intermedio":

Mocquot P. y Moricard R. Compte-rendu de Gyn. et d'Obst. 27: 503. 1938. Moricard R. y F. Rev. franc. de Gyn. pág. 263. 1941.

Moricard R., Moricard F. y Gothié S. Ann d'endocr. 3, fig. 8. págs. 136. 1942.

Moricard F. "Hormonologie Sexuelle Humaine". Fig. 77, 189, Masson. 1943. 
tos fáciles, para caracterizar la acción progestínica sobre un útero foliculinizado.

Realmente el estado foliculínico intermedio es equivalente al estado foliculoluteínico de glucógeno masivo, pues este estado corresponde muy habitualmente a la presencia de un cuerpo amarillo activo. La carga masiva en glucógeno es uno de los primeros efectos característicos de la acción de la progesterona sobre un útero foliculinizado. Las condiciones de esta carga en glucógeno parecen ser diferentes en el Macaccus Rhesus y en la mujer. Es sabido que los frotis vaginales son igualmente diferentes (Shorr) entre la mujer y el Macaccus Rhesus.

b) Estado foliculoluteinico con edema superficial del corion, a los veinte dias, nidación posible: los núcleos se han vuelto esféricos y retornan a la base de cada célula epitelial por razón de las modificaciones del aparato de Golgi; el glucógeno en pequeña cantidad y aun ausente de ciertos tubos glandulares; hay un comienzo de diferenciación de las espinas conjuntivas y de las arteriolas espirales. La diferenciación de las ateriolas parece ser más precoz que la de las espinas. Funck-Brentano, F. Moricard y Rivas han practicado un estudio biométrico del edema. Se puede provocar artificialmente la formación de este edema, el cual se logra bien diferenciado alrededor del sexto dia después de la inyección de $100 \mathrm{mgm}$. de progesterona sobre un útero previamente foliculinizado por la acción de $30 \mathrm{mgm}$. de benzoato de estradiol; este edema no es, pues, un criterio de sobrecarga estrogénica como erróneamente lo han interpretado ciertos autores: es un fenómeno fisiológico muy factiblemente en relación con la niđación del huevo.

c) Estado foliculoluteinico con arteriolas espirales y espinas conjuntivas, pseudogestación, a los veinticuatro y veintiséis dias: si la toma es suficiente se reconocen las arteriolas espirales en la presencia de muchas secciones transversales contiguas situadas en la parte superficial del corion. Se reconocen las espinas conjuntivas en la presencia de varias puntitas conjuntivas (en rojo al Van Gieson) que deforman la pared del tubo glandular y sobre la cual se fija una "borla" o racimo de células cilíndricas. El glucógeno está en excreción en la luz de los tubos glandulares. I.a coloración al muco-carmin es positiva en la mayor parte de los tubos. La presencia de arteriolas espirales y espinas conjuntivas son dos tests equivalentes a las modificaciones conjuntivas 
pseudo-gestativas observadas sobre la coneja en el llamado "encaje uterino" de Bouin y Ancel.

La diferenciación de las arteriolas espirales puede ser obtenida sobre un útero foliculinizado sometido después de seis días a una acción progestínica cristalina de $200 \mathrm{mgm}$. en inyección única. La diferenciación de las espinas parece necesitar una dosis nıás alta de progesterona, que obre por un tiempo más largo. Estas modificaciones conjuntivas son al parecer imposibles de realizar por la sola acción foliculínica y parecen específicas de un equilibrio estradiol-progesterona. La especificidad de efecto de este equilibrio es la base de la exploración cito-hormonal de la fase luteal.

d) Estado foliculoluteinico con transformación deciduiforme, a los veintiséis dias: estado globuloso de las células del corion. las cuales contienen glucógeno en pequeña cantidad. Existe en este período una actividad secretora considerable, con gran cantidad de glucógeno en la luz de las glándulas, formación de muy numerosas espinas conjuntivas y la aparición extensa de la transformación decidual periarteriolar puede permitir sospechar la nidación de un huevo en la mucosa uterina, o una sobrecarga luteal por quiste luteínico o excepcionalmente por tumor ovárico endocrino.

e) Estado foliculoluteínico premenstrual. Invasión de células mononucleadas y también de polinucleares a la mucosa, a los veintiséis $y$ veintisiete dias: en ciertos ciclos. el glucógeno puede desaparecer casi completamente en período premenstrual; cuando la menstruación sobreviene (un día) hay una disociación por las hemorragias del corion alrededor de los tubos glandulares. Modificaciones vaginales acompañan la fase luteal; éstas son esencialmente el plegamiento o doblamiento de las células abundantemente descamadas en grumos, con disminución del índice ácidofílico. Estas modificaciones vaginales no son específicas de un equilibrio estradiol-progesterona y pueden ser observados desde que haya un descenso de la acción estrogénica. Es por esta razón que los frotis vaginales no permiten la exploración de la función liteal.

\section{III- - INTERPRETACION DE LA EXPLORACION CITO-HORMO- NAL UTERINA EN LA FASE LUTEAL}

Conociendo el determinismo hormonal de una prueba uterina específica de la acción progestínica (glucógeno, espinas, arterio- 
las espirales) se puede en presencia de una de estas pruebas remontarse a la causa y afirmar al menos que la acción equilibrada del estrógeno y la progesterona existen sobre este útero. En la práctica la realización de una biopsia del fondo uterino con la cureta de Novak o de Cotte, tres o seis días antes del comienzo de la mestruación, permite afirmar que hay o que ha habido un equilibrio foliculoluteínico. Al presente en la Clínica Ginecológica del Hospital Broca se han estudiado más de 6.000 biopsias endouterinas.

a) Resultados positivos: al observar la presencia de espinas conjuntivas y de arteriolas espirales, la existencia de una acción progestínica parece cierta. La presencia en período premenstrual ce una carga masiva en glucógeno parece indicar formación tardía del cuerpo amarillo en el ciclo. El edema superficial de nidación es de interpretación más difícil, pues los estrógenos inyectacos solo pueden provocar un cierto edema del corion. El edema no puede ser, pues, un test específico del equilibrio estradiol-progesterona, pero este enorme edema de la parte superficial del cosion no traduce tampoco una sobrecarga estrogénica y no puede ser un test de hiperfoliculinemia. La presencia de mitosis no permite diferenciar la acción foliculinica de la progestínica. En el momento actual no es posible interpretar el determinismo hormonal de las capas nucleares múltiples ni de las invaginaciones tubulares sobre las cuales hemos insistido en otras publicaciones.

b) Resultados negativos: la ausencia de diferenciación de la carga en glucógeno o de las espinas conjuntivas y las arteriolas espirales no debe ser siempre interpretada como resultado de una ausencia de formación del cuerpo amarillo. En efecto, puede existir un cuerpo amarillo que no secrete progesterona; esto parece demostrado en la rata y en la coneja. Esta anomalía funcional existe, pues, probablemente también en la patología endocrina de la mujer. Pero sobre todo para que la progesterona marque sus efectos sobre el útero, es preciso que éste sufra una foliculinización anterior y real; en fin, es preciso que no exista un cierto grado de fibrosis del corion como la que se observa en las metrosis de receptividad (Moricard).

El útero puede así no responder a una acción progestínica normal; se puede igualmente concebir una destrucción de la progesterona circulante; esta hormona al ser destruída en el hígado no podría obrar sobre el útero. La ausencia del glucógeno o de 
las espinas conjuntivas y arteriolas espirales no permite, pues, afirmar la ausencia de una acción progestínica, en este caso sólo se puede presumir de una anomalía de la función luteal. Es en este caso que el estudio comparado de biopsias y eliminación del pregnandiol urinario toma una importancia singular. El resultado positivo de una eliminación de pregnandiol premenstrual permite interpretar el resultado negativo de una biopsia; por otra parte, esto es bastante raro. En la interpretación de las biopsias endo-uterinas en la fase luteal, el estudio comparativo de los frotis nos parece ser un trabajo largo y bien inútil. En los casos en que hay aparentes disociaciones entre las dos técnicas, frecuentemente lo que existe en realidad es un error de técnica en la toma de la biopsia, que ha sido hecha no del fondo uterino sino cie la parte inferior del útero. Puede suceder también que haya un error en la interpretación de los frotis, pues se desconoce a menudo que el receptor vaginal es más sensible que el uterino a la acción estrogénica. Por otra parte la receptividad vaginal puecie ser anormal. Así, en 1936, Moricard, al describir las metrosis ce receptividad, señaló el hecho siguiente: madame $\mathrm{Z}$ : cuello pequeño, cúpula vaginal $2.5 \mathrm{cms}$. de distensión. Después del tratamiento con estrógeno, $128 \mathrm{mgm}$. de benzonato de estradiol, la cistensión de la parte inferior de la vagina era de $3,5 \mathrm{cms}$. La cúpula vaginal no se había modificado. Esto muestra el orden de variaciones de la receptividad local a la acción estrogénica.

Cotte ha dado últimamente ejemplos muy interesantes de disociación entre la morfogénesis vaginal y uterina. Es preciso pues, tener en cuenta las anomalías de receptividad vaginal en la interpretación de los frotis vaginales.

\section{CONCLUSION}

En la exploración de la fase luteal:

a) El frotis vaginal es un trabajo largo e inútil, pues no existe en ellos pruebas morfológicas específicas de la acción progestínica (plegamientos celulares). Se confunde muy a menudo una caída de la acción estrogénica con la aparición de la acción progestínica.

b) La dosificación urinaria del pregnandiol necesita una toma simple, pero el dosaje en sí es una técnica delicada que no puede ser actualmente preconizada como método de rutina en la práctica ginecológica corriente. 
c) La biopsia endouterina es una toma un tanto delicada pero sin peligro, gracias a los antibióticos. La lectura anatomopatológica de las biopsias es bastante simple y rápida. Ciertas pruebas son específicas de la acción foliculoluteínica, es sobre esta base que desde 1936 se practica en la Clínica Ginecológica de la Universidad de París en la consulta de esterilidad.

Si dos exámenes pueden ser ejecutados simultáneamente es preciso dar la preferencia a la biopsia endouterina asociada a la dosificación del pregnandiol. Ninguno de los procedimientos preconizados al presente permite un estudio cuantitativo de la fase luteal. El único hecho real es poder reconocer en una mujer la existencia de la función luteal en el curso del ciclo menstrual es decir, de poder concluir que la función endocrina ovariana foliculoteínica parece ser normal.

En la práctica la base de la exploración de la función luteal es la biopsia endouterina, la cual permite reconocer modificaciones específicas de acción foliculoluteínica.

\section{BIBLIOGRAFIA}

(Se encontrará una bibliografía detallada en "Exploration Cytohormonale" nueva clasificación, volumen del reporte del Primer Congreso Internacional de Biología Clínica, por R. y F. Moricard, p. 139. París. 1948. Señalamos aquí algunas referencias de base, y más especialmente las publicaciones posteriores a 1948 y anteriores a 1953).

1. ALEEAUX-FERNeT, M. y BUSSER, F.- Sem. des Hop. p. 4, 480. 1950.

2. ASCHEIM, S. Z.-Fur Gynakol.: 39, 65. 1915.

3. ASTWOOD, E. B. et JONES, G. E. S.- J. Biol. chem. 137, 397. 1941.

4. BORT'H, R.- Colloquia on Endocrinology II. 45. 1952. Churchill, Ltd.

5. BRADBURY, J. et BROWN, W. E.- J. of Clin. Endo., II. 267. 1951.

6. BROKMANN, H. and SCHODDER, M.- Ber dtsh. chem. ges. 74, 73. 1941.

7. BUTENADT, A. and FLEISHER, G.- Ber dtsh. chem. ges. 68, 2.094. 1935.

8. CLAUBERG, G.- Die Weiblichen Sexualhormone, Berlin, 1933.

9. FERIN, J.- Acta Med. Belgi. 3, 360. 1948.

Ann. d'endo., II. 179, 1950.

Eull. de la Soc. de Gyn. et d'Obs. 3, 553. 1951.

10. FUNCK-BRENTANO, F. MORICARD, F. et RIVAS, M.- Bull de la Soc. de Gyn. et d'Obs. lg. franc. 3, 344. 1951.

11. FUNCK-BRENTANO, P. MORICARD, R. et ROBERT, H. B.- Bull. de la Soc. de Gyn. et d'Obs. lg. franc. 3, 381. 1951.

12. HUBERD, D.- Ch. Journ. 41, 609. 1947.

13. JAYLE, M.- Ann. Endo. 9, 391. 1948. Soc. de Gyn. et d'Obs. París, nov. 1953.

14. JAYLE, M. VALlin, Y. et CREPY, O.- Ann. Endo. 10, 363, 1949. 
15. KAUFMANN, K.- Klin. Wochen No 6, 117, 1953.

16. MASTER, W. et MAGALLON, D.- J. of Clin. Endo. 10, 349. 1950.

17. MOCQUOT, P. et MORICARD, R. C. R.-Soc. Gyn. et d'Obs., 27, 503. 1938.

18. MORICARD, F.- Hormonologie Sexuelle Humaine, Masson, 1943.

19. MORICARD, R.- Rev. fr. de Gyn., 31, 740. 1936.

Congres franc. de Gyn., Rev. franc. Gyn. 29: p. 759. 1934.

L'exploration cytohormonale en Gynecologie, Rev. franc. de Gyn. 31: 740, 1936.

Bull. Soc. Gyn. et d'Obs. 25, 422, 426, 432, 435, 526, 529, $533,1936$.

20. MORICARD, R., MORICARD, F. et GOTHIE S.- No 3: 125. 1942.

21. MORICARD, R. et MORICARD, F.- Congres gyn. Londres. 1949.

22. MOUKAYE et CHAMPY, C.- Gyn. et Obs. 404. 1921.

23. NOYES, R W., HERTIC, A. T. Et ROCK. J. of Fert. and Ster., 1: p. 3, 1950.

21. PAPANiCOlaOU, F. et SHORR.- Am. J. Obs. Gyn., 31: 806. 1936.

The epithelia of woman's reproductive organs, N. Y. 1948.

24. PAPANICOLAOU, F. et SHORRE.- Am. S. Obs. and Gyn., 31: 806. 1936.

25. PROUST, R., MORICARD, R. et PALMER, R.- Bull et mem. Soc. nat. de chir. 61: 1100. 193.

25. PUNDEL, J. P.- Frottis vaginaux endocriniens Masson. 1952.

26. SALMON, U. J. et FRANK, R. T.- 33: 612, 1936.

27. SCHOKAFRT, A. et FERIN, J.- Brux. med. 4. 1940.

TRIQUET. Etude comparee Biopsies d'endometre et frottis vaginaux, these, Lille, 1952.

29. VENNING, E. M. et BROWN.- Endocrinology 21: 711. 1937.

30. WATteVille, H. de BORTH, R. et GSELL, M.- J. of Clin. Endo., 8: 982, 992. 1948.

31. WAtteville, H. de J.- Cl. Endo. II: 251. 1951. 